\title{
Effects of Low-level Laser Therapy on Muscle Repair in Rats with Chronic Alcohol Intake
}

\author{
Isabel Cristina Cespedes ${ }^{1}$, Livia Assis ${ }^{12} 2^{*}$, Rafael Moraes Thomaz ${ }^{1}$, Carlos Eduardo \\ Panfilio $^{1}$, Luis Gonçalves ${ }^{1}$, Ana Claudia Renno Muniz ${ }^{1}$ \\ ${ }^{1}$ Universidade Federal de São Paulo - Departamento de Biociências, Santos, São Paulo, Brazil; ${ }^{2}$ Universidade \\ Brasil, São Paulo, São Paulo, Brazil
}

\begin{abstract}
This study aimed to investigate the effects of low-level laser therapy (LLLT) on muscle repair in rats with chronic alcohol intake. Thirty male Wistar rats were distributed into three groups: injured tibialis anterior (TA) muscle without treatment (IC); chronic ingestion of alcohol plus injured TA muscle (AI); and chronic ingestion of alcohol plus injured TA plus LLLT (AIL). Each group was divided into two different subgroups, and rats were sacrificed on days 3 and 7 post-injury. Morphological features in the injured areas were similar with or without alcohol intake (IC and AI); however, LLLT promoted a decrease in the number of inflammatory cells and destroyed zones, as well as improved tissue organization (AIL). In general, alcohol intake caused a decrease in myogenic regulatory factors (MyoD and myogenin) and vascular endothelial growth factor in the AI group. Moreover, LLLT promoted recovery of these factors to the same level as in animals without alcohol intake (IC and AIL). LLLT was able to increase the expression of myogenic and vascular growth factors and stimulate skeletal muscle regeneration in rats with chronic alcohol intake.
\end{abstract}

Keywords: Low-level laser therapy; alcohol intake; muscle repair

*Author for correspondence: livinha_fisio@yahoo.com.br 


\section{INTRODUCTION}

Excessive consumption of alcohol is associated with different diseases of the brain, as well as other vital organs such as the heart, lung, liver, pancreas, and kidneys ${ }^{1}$. The immune system may be severely affected by the effects of alcohol on the different cellular components of the innate and/or adaptive system, with disruption of signaling pathways involved in initiation and control of the inflammatory response ${ }^{2-4}$. Studies have shown that in infectious conditions, alcohol abuse promotes an increase in bacterial load, which exacerbates injury. Moreover, the oxidant stress present in chronic ethanol ingestion contributes to the generation of proinflammatory cytokines, thus altering the tissue repair process ${ }^{4}$. The musculoskeletal system is also affected by chronic alcohol ingestion, with muscle atrophy, decreased fiber diameter, and muscle protein expression, and an increase in the number of myopathic lesions ${ }^{5,6}$.

Innovative clinical approaches to accelerate muscle metabolism and repair damaged muscle tissue in the presence of disease have been developed, and include lowintensity pulsed ultrasound (LIPUS), pulsed electromagnetic field, and extracorporeal shock wave modalities ${ }^{7-9}$. Low-level laser therapy (LLLT) is also a common modality used to treat musculoskeletal condition ${ }^{10,11}$. Many studies have shown that LLLT is effective in reducing post-injury inflammatory processes and myonecrosis, accelerating the proliferation of satellite cells, and stimulating the formation of new blood vessels ${ }^{8,9,12}$. Furthermore, a series of studies have demonstrated that LLLT has positive effects on muscle tissue and muscle repair. Rodrigues et al. ${ }^{13}$ demonstrated that $660-\mathrm{nm}$ laser at $5 \mathrm{~J} / \mathrm{cm}^{2}$ promoted upregulation of MyoD and myogenin mRNA expression after laser irradiation 7 days after injury. Our group compared the effects of LIPUS and 830-nm laser therapy at $50 \mathrm{~J} / \mathrm{cm}^{2}$, and observed that laser-irradiated animals had a smaller necrotic area and better tissue organization at the site of the injury than did LIPUS-treated animals ${ }^{7}$.

Despite the positive effects of LLLT on tissue regeneration, the mechanism by which such therapy acts on muscle tissues is not fully understood, and no reports were found investigating the effects of LLLT on muscle repair in alcoholics. Moreover, the literature reports a wide range of wavelengths and fluencies used by different authors, which makes it difficult to compare published results and determine an optimal treatment protocol. However, since laser therapy is widely used to accelerate tissue healing, the aim of this study was to verify the effects of 808-nm laser therapy on injured skeletal muscle after cryolesioning, using histopathology, morphometry, and immunohistochemistry, in animals subjected to chronic alcohol intake.

\section{MATERIALS AND METHODS}

\section{Experimental groups}

Thirty Wistar male rats (weighing $240 \pm 20$ g) were used in the current study. They were maintained under controlled temperature $\left(22 \pm 2^{\circ} \mathrm{C}\right)$, with light-dark periods of 12 $\mathrm{h}$, and with free access to water and a commercial diet. All animal handling and surgical procedures were strictly conducted according to the Guiding Principles for the Care and Use of Laboratory Animals. This study was approved by the Committee of the Federal University of São Paulo - 0383/2012. Animals were randomly distributed into three groups: injured tibialis anterior muscle without treatment (IC: injury control); chronic ingestion of alcohol plus injured tibialis anterior muscle without treatment (AI: alcohol plus injury); and chronic ingestion of alcohol plus injured tibialis anterior muscle submitted to laser irradiation treatment (AIL: alcohol plus 
injury plus LLLT). Each group was divided into two different subgroups $(\mathrm{n}=5)$, and the rats were sacrificed on days 3 and 7 post-injury.

\section{Chronic ingestion of alcohol}

For chronic administration of alcohol, the paradigm used two bottles with intermittent access. A bottle of water and a bottle containing $20 \%$ alcohol with no added sugar were continuously offered to the animals during three weekly sessions with $24 \mathrm{~h}$ of exposure (Mondays, Wednesdays, and Fridays), for a total of 20 sessions. A bottle with $20 \%$ alcohol solution and another bottle with water were offered to the animals on a Monday, after a week of habituation. This pattern was maintained on the subsequent Wednesday and Friday. On days when the bottle of alcohol was removed, a second bottle of water was made available. The animals had unlimited access to two water bottles on weekends. The position of the alcohol bottle was alternated every session to avoid consumption by place preference. The bottles were weighed before and $24 \mathrm{~h}$ after being offered to the animals to control the consumption of the solutions. Alcohol solutions were prepared with tap water from absolute ethanol $99.5 \%(\mathrm{v} / \mathrm{v})$ (Labsynth Products Laboratories Ltd., Diadema, SP, Brazil) ${ }^{14}$. The alcohol solution was presented in 250-ml graduated glass cylinders with stainless-steel drinking spouts wrapped in rubber. Alcohol intake was analyzed three times a week at each offer of a new bottle and the body weight was assessed at the beginning and end of treatment to obtain an average weight during treatment.

\section{Freezing muscle injury (cryolesion)}

A surgical procedure (cryolesion) on the tibialis anterior muscle (TA) was performed based on a description by Miyabara et al. ${ }^{15}$ under anesthesia with $40 \mathrm{mg} / \mathrm{kg}$ ketamine (Dopalen, Vetbrands; São Paulo, Brazil) and $20 \mathrm{mg} / \mathrm{kg}$ xylazine (Anasedan, Vetbrands). After anesthesia, the skin around the right TA muscle was shaved and cleaned. Then, a 1-cm transverse skin incision was made to expose the middle muscle. A rectangular iron bar $(40 \times 20 \mathrm{~mm})$ frozen in liquid nitrogen was then applied for 10 $\mathrm{s}$ to the center of the muscle. The procedure was repeated twice consecutively, with a time interval of $30 \mathrm{~s}$. The skin was then sutured. The right TA muscle was chosen because it is superficial, making surgery easy. After surgery, the animals were housed in single plastic cages in a room with controlled environmental conditions, and were fed rat chow and water ad libitum.

\section{LLLT Protocol}

LLLT was performed using a gallium-aluminum-arsenide (GaAlAs) diode laser (PHOTON LASER II, DMC ${ }^{\circledR}$ Equipamentos Ltda., SP, São Carlos, Brazil), with the following parameters: continuous radiation mode, $808-\mathrm{nm}$ wavelength, $30 \mathrm{~mW}$ power output, $47 \mathrm{~s}$ irradiation time, $0.00785 \mathrm{~cm}^{2}$ spot area, dose $180 \mathrm{~J} / \mathrm{cm}^{2}$, irradiance 3.8 $\mathrm{W} / \mathrm{cm}^{2}$, and $1.4 \mathrm{~J}$ total energy per point ${ }^{10}$. The laser was applied over one point at the middle of the right TA muscle (lesion area). LLLT was performed daily and at the same time for 3 or 7 consecutive days, with the first application immediately after skin suturing. Laser was applied by contact technique, with the optical fiber kept perpendicular to the skin. The animals were handled gently, and LLLT did not produce any painful sensation or distress to the animals. 


\section{Histopathological analysis}

Muscles obtained from all experimental groups were washed immediately with saline and each specimen was fixed in 10\% buffered formalin (Merck, Darmstadt, Germany) for $24 \mathrm{~h}$, followed by dehydration in a graded series of ethanol and embedding in paraffin. In the longitudinal axis of the muscle, thin sections $(5 \mu \mathrm{m})$ were prepared using a microtome (Leica Microsystems SP 1600, Nussloch, Germany). Five sections of each specimen were stained with hematoxylin and eosin (HE stain, Merck) and examined using light microscopy (Leica Microsystems AG, Wetzlar, Germany). Histopathological evaluation was performed by an experienced pathologist (DAR) (blinded to the treatment) and was carried out under a light microscope (Olympus, Optical Co. Ltd, Tokyo, Japan) at 40× magnification. The qualitative analysis considered any changes at the injury site, such as presence of an inflammatory process, granulation tissue, necrosis area, focal or diffuse myofibrillary degeneration, and tissue structure alterations ${ }^{7}$.

\section{Morphometry of the injured area}

For morphometric evaluation, one histological cross section of each TA muscle located in the central region of injury was chosen to measure the area using software for morphometry (Axiovision 3.0.6 SP4, Carl Zeiss, Jena, Germany). Images were used to reconstruct the total muscle cross-sectional area, allowing the identification and measurement of the injured area. A double-blind procedure was used for muscle cross section image selection and injured muscle area measurements.

\section{Immunohistochemistry}

Serial longitudinal muscle sections of $4 \mu \mathrm{m}$ were deparaffinized in xylene and rehydrated in graded ethanol, then pretreated by microwave (Brastemp, SP, Brazil) with $10 \mathrm{mM}$ citric acid buffer $(\mathrm{pH}=6)$ for three cycles of 5 min each at $850 \mathrm{~W}$ for antigen retrieval. The material was preincubated with $0.3 \%$ hydrogen peroxide in phosphate buffered saline (PBS) for 5 min for inactivation of endogenous peroxidase, and then blocked with 5\% normal goat serum in PBS solution for $10 \mathrm{~min}$. The specimens were then incubated with anti-MyoD, anti-myogenin, and anti-vascular endothelial growth factor (VEGF) antibodies (Santa Cruz Biotechnology, USA) at a concentration of $1: 100$. Incubation was carried out overnight at $4^{\circ} \mathrm{C}$ in a refrigerator and followed by two washes in PBS for $10 \mathrm{~min}$. The sections were then incubated with biotin-conjugated secondary antibody (anti-rabbit $\mathrm{IgG}$ ) (Vector Laboratories, Burlingame, CA, USA) at a concentration of 1:200 in PBS for $1 \mathrm{~h}$. The sections were washed twice with PBS before the application of preformed avidin-biotin complex conjugated to peroxidase (Vector Laboratories) for $45 \mathrm{~min}$. The bound complexes were visualized by the application of a $0.05 \%$ solution of 3-3'-diaminobenzidine and counterstained with Harris hematoxylin. For control studies of antibodies, the serial sections were treated with rabbit IgG (Vector Laboratories) at a concentration of 1:200 in place of the primary antibody. Furthermore, internal positive controls were performed with each staining bath. Digital images of the 100x magnification were captured by optical microscopy (Leica Microsystems). Two experienced observers (LA and RT) performed the analyses in a blinded manner. 


\section{Statistics}

Data are expressed as the mean \pm standard error of the mean (SEM). The ShapiroWilk and Levene tests were applied to evaluate normality and homogeneity of the results, respectively. For the variables that exhibited normal distribution, comparisons between experimental groups were performed by analysis of variance (one-way ANOVA), and the Tukey post-test was used to compare individual groups. For the variables that exhibited non-normal distribution, the Kruskal-Wallis test was used. A $P$ value $<0.05$ was considered significant. All analyses were performed using a GraphPad Prism 6.0 (GraphPad Software, San Diego CA, USA).

\section{RESULTS}

\section{Intake alcohol}

The average consumption of alcohol during the treatment period was $15 \mathrm{~g} / \mathrm{kg} / 24 \mathrm{~h}$. Morphological analysis

Representative images of TA muscle sections are shown in Figure 1. On morphological analysis at 3 days post-injury, IC and AI showed severe alterations, with acute and extensive myofibrillar degeneration, destroyed zones, interstitial edema, and an intense inflammatory reaction composed mainly of mononuclear cells. AIL had findings similar to IC and AI, but with less inflammatory infiltrate.

At 7 days post-injury, destroyed zones, inflammatory cell infiltrate, fascicular disorganization, tissue necrosis, and abundant extracellular matrix deposition were observed. AIL showed minor areas of destroyed zones, less inflammatory cell infiltrate, and better tissue organization in the area corresponding to the cryolesion site.

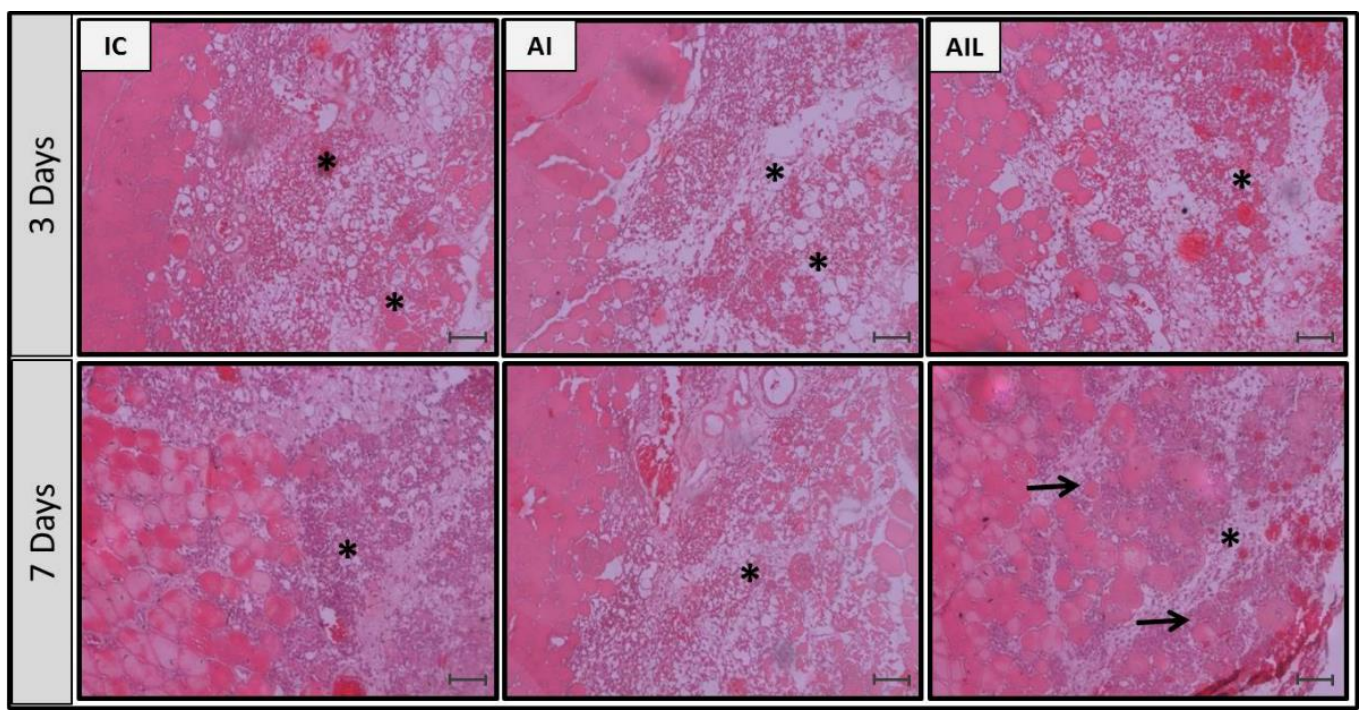

Figure 1 - Morphological analysis of middle TA muscle cross sections 3 and 7 days post-injury. Injured TA muscle without treatment (IC); chronic ingestion of alcohol plus injured TA muscle without treatment (AI); chronic ingestion of alcohol plus injured TA muscle submitted to laser irradiation treatment (AIL). Asterisks indicate inflammatory infiltrate, and arrows indicate better tissue organization. HE stain, Magnification 20x 


\section{Muscle injury area}

There was no significant difference in the TA muscle injury area among the groups, in both experimental periods after surgery ( $p>0.05$; Fig. 2 A and B).

A

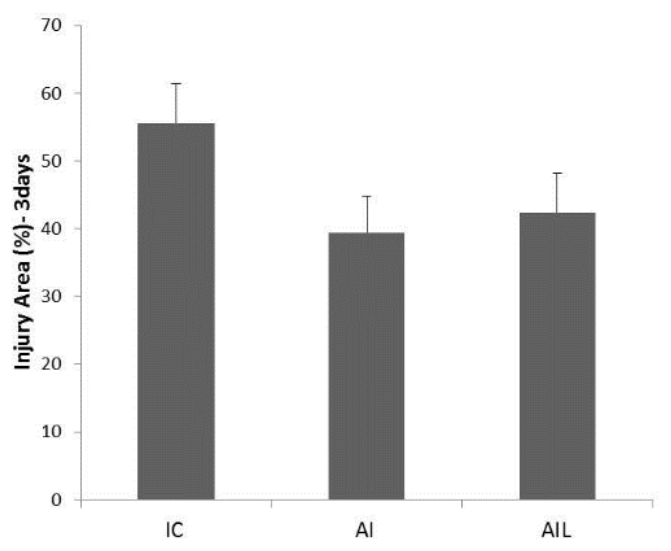

B

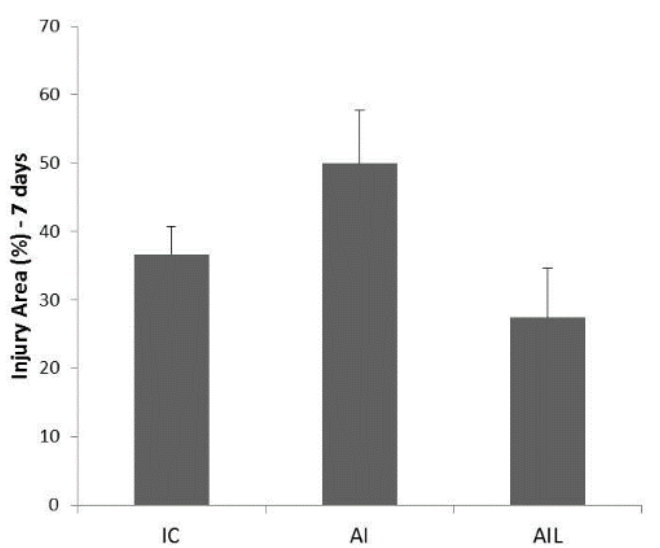

Figure 2 - Injured area. A) 3 days post-injury; B) 7 days post-injury. Injured tibialis anterior muscle without treatment (IC); chronic ingestion of alcohol plus injured TA muscle without treatment (AI); chronic ingestion of alcohol plus injured TA muscle submitted to laser irradiation treatment (AIL).

\section{Immunohistochemistry}

\section{MyoD expression}

Immunohistochemical data for MyoD are summarized in Figure 3. Immunostaining was detected in the nucleus of muscle cells and circumjacent areas. On day 3 postsurgery, moderate MyoD immunoexpression was observed in all experimental groups. On day 7 post-surgery, moderate MyoD immunoexpression was observed in the muscle fibers of IC and AIL. However, AI presented decreased MyoD immunoexpression at the site of the injury.

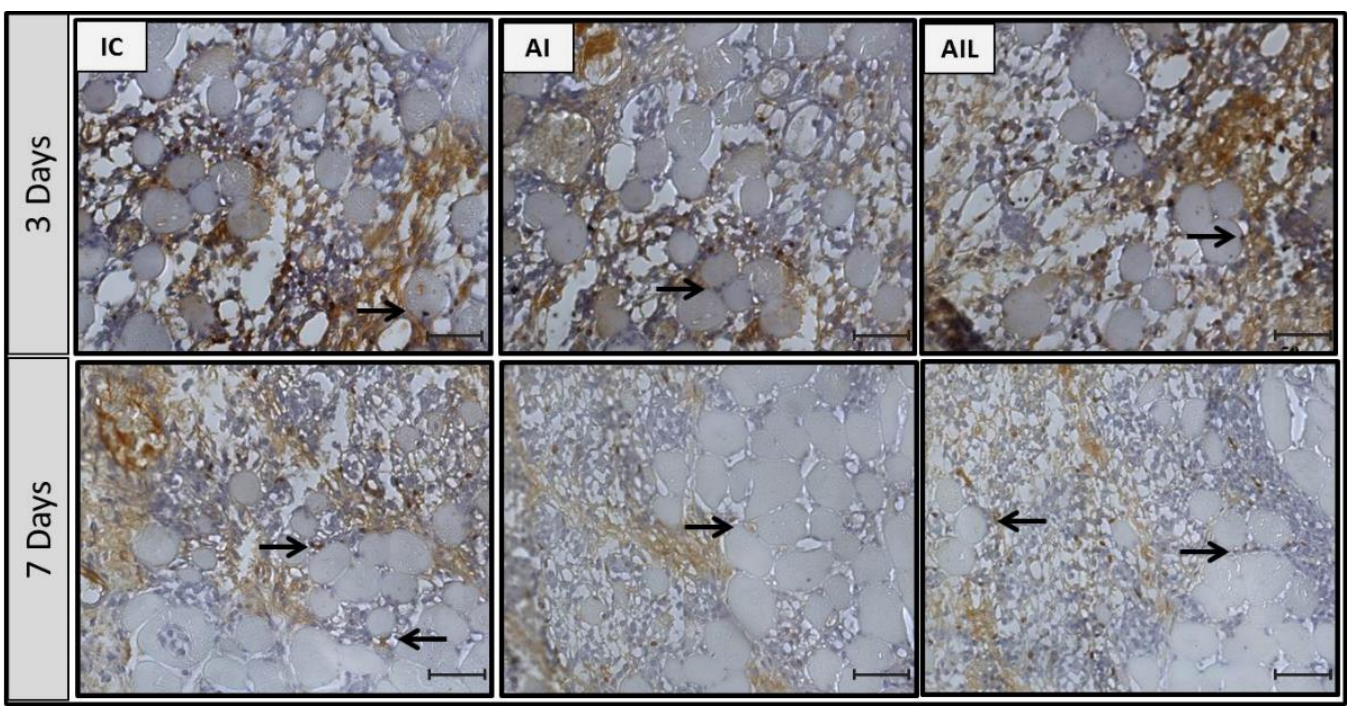

Figure 3 - Immunohistochemistry for MyoD 3 and 7 days post-injury. Injured TA muscle without treatment (IC); chronic ingestion of alcohol plus injured TA muscle without treatment (AI); chronic ingestion of alcohol plus injured TA muscle submitted to laser irradiation treatment (AIL). Magnification 40x 


\section{Myogenin expression}

Immunohistochemical data for myogenin are demonstrated in Figure 4. Immunostaining was detected in the nucleus of muscle cells and circumjacent areas. On day 3 post-surgery, moderate myogenin immunoexpression was observed in IC. However, AI and AIL presented slight myogenin immunoexpression at the site of the injury. On day 7 post-surgery, intense myogenin immunoexpression was observed in the muscle fibers of IC and AIL. In contrast, AI presented moderate myogenin immunoexpression at the site of the injury.

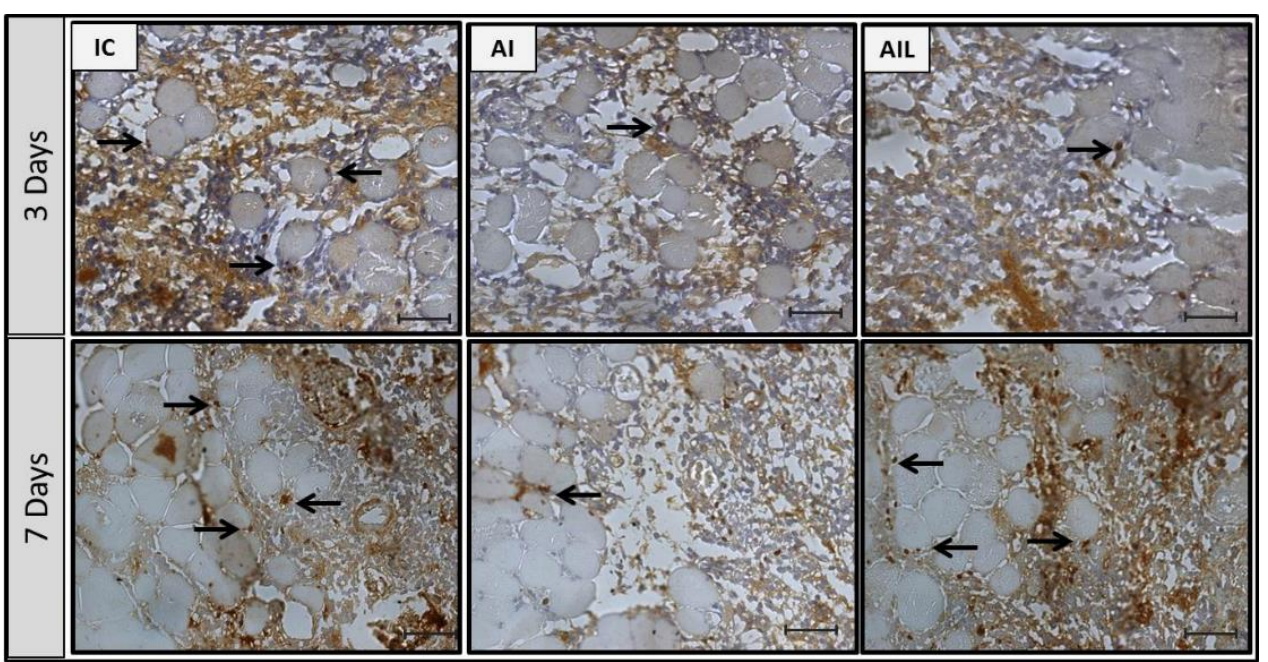

Figure 4 - Immunohistochemistry for Myogenin 3 and 7 days post-injury. Injured TA muscle without treatment (IC); chronic ingestion of alcohol plus injured TA muscle without treatment (AI); chronic ingestion of alcohol plus injured TA muscle submitted to laser irradiation treatment (AIL). Magnification 40x

\section{VEGF expression}

VEGF expression was detected in the cytoplasm of inflammatory and muscle cells. On day 3 post-surgery, moderate VEGF immunoexpression was observed in IC and AIL. Interestingly, AI presented mild expression for this immunomarker. On day 7 postsurgery, intense VEGF immunoexpression was observed in IC and AIL. By contrast, AI presented moderate VEGF immunoexpression at the site of the injury (Fig. 5). 


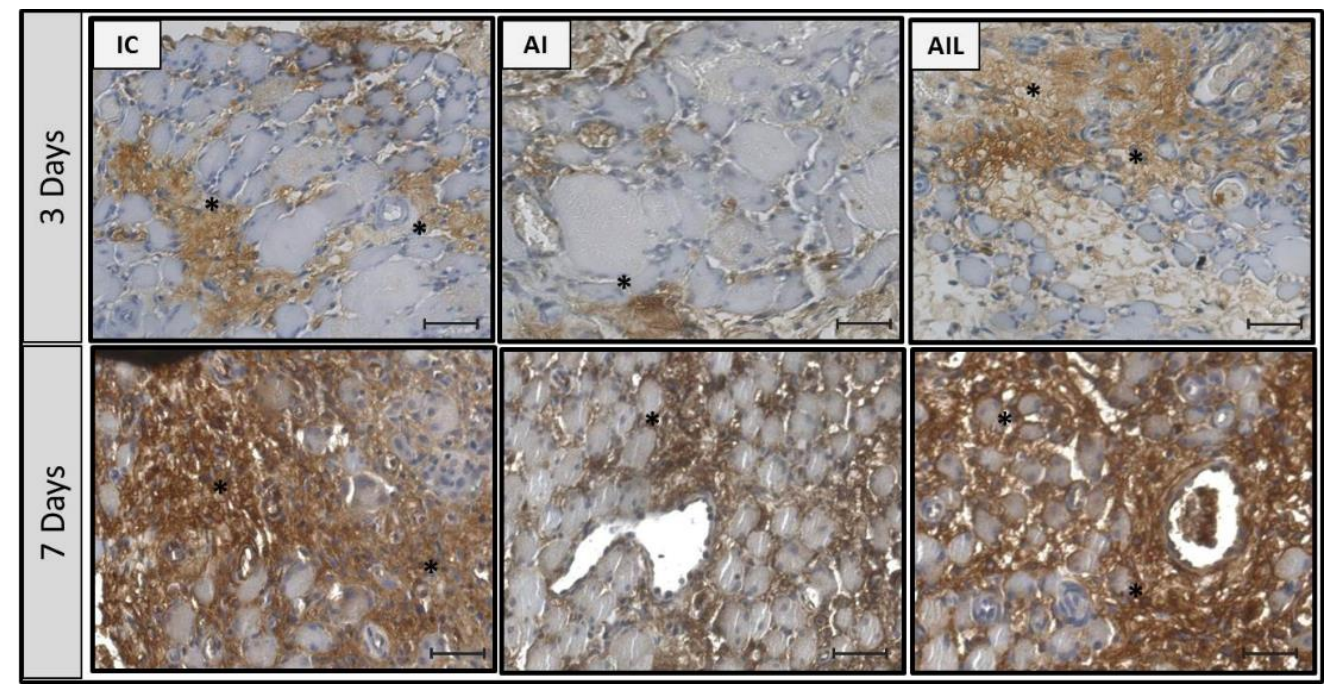

Figure 5 - Immunohistochemistry for VEGF 3 and 7 days after injury. Injured TA muscle without treatment (IC); chronic ingestion of alcohol plus injured TA muscle without treatment (AI); chronic ingestion of alcohol plus injured TA muscle submitted to laser irradiation treatment (AIL). Magnification 40x

\section{DISCUSSION}

This study aimed to evaluate the effects of LLLT on muscle repair processes in rats with chronic alcohol intake. The main findings were that the laser-treated animals showed positive effects on the process of tissue repair and produced greater MyoD, myogenin, and VEGF immunoexpression compared to the untreated group (AI).

It is known that chronic alcohol consumption leads to progressive alterations in the musculoskeletal system, such as muscle atrophy and decreased skeletal muscle repair $^{16}$. This process may be related to alcohol-induced apoptosis of skeletal muscle, suppressed release of proinflammatory cytokines, diminished neutrophil recruitment, and altered phagocytic and chemotactic functions ${ }^{16,17}$.

The dose and timing of ethanol exposure can induce variable effects on immune function and on organ tissues. In this study, the animals submitted to the two-bottle paradigm had an average intake of $15 \mathrm{~g} / \mathrm{kg} / 24 \mathrm{~h}$. Multiple in vivo and in vitro studies showed that acute ethanol intake inhibits the production of proinflammatory cytokines in response to a variety of microbial compounds. In animal studies in which ethanol was administered by intraperitoneal injection or oral gavage, the inhibitory effect was consistently observed with doses of 0.8 to $6 \mathrm{~g} / \mathrm{kg}$ body weight, corresponding to moderate and binge drinking patterns, respectively ${ }^{18}$. In another review, Messingham et al. ${ }^{19}$ reported studies in which very low ethanol concentrations (acute intake of 0.5 $\mathrm{g} / \mathrm{kg} /$ day for 5 days, or chronic intake of $0.43 \mathrm{~g} / \mathrm{kg} /$ day for 28 days) showed a stimulatory effect on cellular immune responses. However, a high acute $(6 \mathrm{~g} / \mathrm{kg} / \mathrm{day}, 5$ days) or chronic (12 g/kg/day, 28 days) dose of ethanol induced significant suppression of immunological parameters. The achieved dose in our study can be considered a moderate chronic pattern.

The histological findings showed that the cryolesion model induced muscle tissue degeneration and an intense inflammatory process in the area of the lesion in all groups. Moreover, the infrared laser-irradiated animals presented reduced inflammatory infiltrate and better tissue organization in both periods evaluated. However, no difference was observed in the morphometric evaluation of the area of injury. The stimulatory effects of LLLT on biological tissues, including muscle tissue, have been demonstrated by many authors ${ }^{9}$. Such findings may reflect the positive 
effects of LLLT on muscle repair, demonstrating its mitogenic potential and capacity for activating satellite and myogenic cells, culminating in better tissue organization at the site of an injury ${ }^{20}$. Moreover, our group had published studies demonstrating a decrease in musculoskeletal area of injury and improvement in tissue organization using the same experimental model ${ }^{10,20,21}$.

Furthermore, immunohistochemistry analysis was used to verify the expression of proteins related to the regulation of skeletal muscle repair (MyoD and myogenin). Both MyoD and myogenin are transcription factors important in myogenesis, as well as conversion of satellite cells into myoblasts and differentiation into myoblasts, which fuse to repair damaged fibers ${ }^{22}$. The results of the present work showed that alcohol intake induced a decrease in MyoD and myogenin expression at 3 and 7 days after cryolesioning. According to Arya et $\mathrm{al}^{23}$, the loss of muscle mass in alcoholic myopathy may reflect inhibition of myogenic cell differentiation into myotubes. The authors demonstrated that ethanol administration during differentiation of the skeletal muscle reduced MyoD and myogenin expression. Moreover, the activation of satellite cells and consequent expression of transcription factors such as MyoD and myogenin are mediated by cytokines released by inflammatory cells ${ }^{22}$. Danna et al. ${ }^{1}$ demonstrated the general phases of muscle regeneration and fibrosis, which are precipitated by a variety of cell types, cytokines, and growth factors. It is known that ethanol alters cytokine levels in a variety of tissues promoting alterations in the inflammatory response ${ }^{24}$. This may explain the apparent decrease in MyoD and myogenin expression in animals exposed to an alcoholic solution in our study. Interestingly, LLLT was able to prevent this downregulation (once the expression for IC and AIL was similar). Some studies have shown the positive effects of LLLT in modulating growth factors and protein expression related to skeletal muscle repair. Brunelli et al. ${ }^{21}$ demonstrated that LLLT was able to increase MyoD and myogenin immunoexpression 7 days after muscle injury in rats. In addition, Assis et al. ${ }^{10}$ observed that LLLT had the potential to increase MyoD and myogenin mRNA expression after laser irradiation. Thus, a higher expression of MyoD and myogenin observed in laser-treated animals led us to infer that LLLT treatment may stimulate satellite cell proliferation, contributing to enhanced muscle repair.

Moreover, ethanol promotes a delay in angiogenesis during tissue healing ${ }^{16}$. In addition, it has been suggested that this effect is mediated by changes in endothelial cell responsiveness to both VEGF and hypoxia of the tissue ${ }^{25}$. VEGF is a signal protein produced by many cell types that exerts multiple effects on the vascular endothelium, including stimulation of endothelial cell proliferation and subsequent connection between new vessels and preexisting circulation ${ }^{26}$. In the current study, decreased expression of VEGF was observed in AI at 3 and 7 days after cryolesioning. Nevertheless, LLLT induced recovery of expression of these factors to the same level as the animal that had no alcohol intake (IC and AIL groups) in both periods evaluated. Studies show positive effects of LLLT in regenerating muscle, with an increase in myotubes, myofibrils, and angiogenesis ${ }^{27,28,29}$. Rodrigues et al. ${ }^{20}$ and Assis et al. ${ }^{8}$ used the same experimental model and found that LLLT caused an increase in the expression of VEGF mRNA after muscle lesioning. Thus, the increased expression of VEGF after laser irradiation may stimulate the formation of new blood vessels, contributing to the acceleration of muscle regeneration in animals with chronic ingestion of alcohol.

Consequently, these data highlight the potential of LLLT to be used as a therapeutic approach to stimulate skeletal muscle repair in compromised conditions, such as chronic alcohol intake. As this study was limited to relatively short-term evaluation of the effects of only one set of laser parameters, information on the long-term performance of this therapy and other laser parameters is needed. 
In conclusion, LLLT was able to increase the expression of myogenic and vascular growth factors, stimulating skeletal muscle regeneration in rats with chronic alcohol intake.

\section{CONFLICT OF INTEREST}

No competing financial interests exist.

\section{ACKNOWLEDGEMENTS}

We thank to Brazilian funding agencies FAPESP for the financial support of this research.

\section{REFERENCES}

1 Danna NR, Beutel BG, Campbell KA, Bosco JA. Therapeutic approaches to skeletal muscle repair and healing. Sports Health. 2014; 6(4): 348-355

2 Cook RT. Alcohol abuse, alcoholism, and damage to the immune system--a review. Alcohol Clin Exp Res. 1998; 22(9): 1927-1942.

3 Szabo G. Consequences of alcohol consumption on host defence. Alcohol Alcohol. 1999; 34(6): 830.

4 Brown LA, Cook RT, Jerrells TR, Kolls JK, Nagy LE, Szabo G, et al. Acute and chronic alcohol abuse modulate immunity. Alcohol Clin Exp Res. 2006; 30(9): 1624-1631

5 Preedy VR, Peters TJ. The effect of chronic ethanol ingestion on protein metabolism in typeI- and type-II-fibre-rich skeletal muscles of the rat. Biochem J. 1988; 254(3): 631-639.

6 Reilly ME, G McKoy D, Mantle TJ, Peters G, Goldspink and VR. Protein and mRNA levels of the myosin heavy chain isoforms Ibeta, IIa, IIx and IIb in type I and type II fibrepredominant rat skeletal muscles in response to chronic alcohol feeding. J Muscle Res Cell Motil. 2000; 21(8): 763-773.

7 Renno AC, Toma RL, Feitosa SM, Fernandes K, Bossini PS, de Oliveira P. Comparative effects of low-intensity pulsed ultrasound and low-level laser therapy on injured skeletal muscle. Photomed Laser Surg. 2011; 29(1): 5-10.

8 Assis L, Moretti AI, Abrahão TB, de Souza HP, Hamblin MR, Parizotto NA. Low-level laser therapy $(808 \mathrm{~nm})$ contributes to muscle regeneration and prevents fibrosis in rat tibialis anterior muscle after cryolesion. Lasers Med Sci. 2013;28(3): 947-955.

9 Alves AN, Fernandes KP, Melo CA, Yamaguchi RY, França CM, Teixeira DF, et al. Modulating effect of low level-laser therapy on fibrosis in the repair process of the tibialis anterior muscle in rats. Lasers Med Sci. 2014; 29(2): 813-821.

10 Assis L, Moretti AI, Abrahão TB, Cury V, Souza HP, Hamblin MR, et al. Low-level laser therapy $(808 \mathrm{~nm})$ reduces inflammatory response and oxidative stress in rat tibialis anterior muscle after cryolesion. Lasers Surg Med. 2012; 44(9): 726-735.

11 Baptista J, Martins MD, Pavesi VC, Bussadori SK, Fernandes KP, Pinto Junior D dos S. Influence of laser photobiomodulation on collagen IV during skeletal muscle tissue remodeling after injury in rats. Photomed Laser Surg. 2011; 29(1): 11-17.

12 Chung H, Dai T, Sharma SK, Huang YY, Carroll JD, Hamblin MR. The nuts and bolts of low-level laser (light) therapy. Ann Biomed Eng. 2012 Feb;40(2): 516-533.

13 Rodrigues NC, Assis L, Fernandes KR, Magri A, Ribeiro DA, Brunelli R, et al. Effects of $660 \mathrm{~nm}$ low-level laser therapy on muscle healing process after cryolesion. J Rehabil Res Dev. 2013; 50(7): 985-996.

14 Simms JA, Steenland P, Medina B, Abernathy KE, Chandler L J, Wise R, et al. Intermittent Access to $20 \%$ Ethanol Induces High Ethanol Consumption in Long-Evans and Wistar Rats Alcohol. Clin Exp Res. 2008; 32(10): 1816-1823.

15 Miyabara EH, Martin JL, Griffin TM, Moriscot AS, Mestril R. Overexpression of inducible $70-\mathrm{kDa}$ heat shock protein in mouse attenuates skeletal muscle damage induced by cryolesioning. Am J Physiol Cell Physiol. 2006; 290(4): C1128-1138. 
16 Hapa O, Cakici H, Gideroğlu K, Ozturan K, Kükner A, Buğdayci G. The effect of ethanol intake on tendon healing: a histological and biomechanical study in a rat model. Arch Orthop Trauma Surg. 2009; 129(12): 1721-1726.

17 Afshar M, Richards S, Mann D, Cross A, Smith GB, Netzer G, et al. Acute immunomodulatory effects of binge alcohol ingestion. Alcohol. 2015; 49(1): 57-64.

18 Goral J, Karavitis J, Kovacs EJ. Exposure-dependent effects of ethanol on the innate immune system. Alcohol. 2008; 42(4): 237-247.

19 Messingham KA, Faunce DE, Kovacs EJ. Alcohol, injury, and cellular immunity. Alcohol. 2002; 28(3):137-49.

20 Rodrigues NC, Brunelli R, Abreu DC, Fernandes K, Parizotto NA, Renno AC. Morphological aspects and Cox-2 expression after exposure to 780-nm laser therapy in injured skeletal muscle: an in vivo study. Braz J Phys Ther. 2014; 18(5): 395-401.

21 Brunelli RM, Rodrigues NC, Ribeiro DA, Fernandes K, Magri A, Assis L, et al. The effects of 780-nm low-level laser therapy on muscle healing process after cryolesion. Lasers Med Sci. 2014; 29(1): 91-96.

22 Järvinen TA, Järvinen M, Kalimo H. Regeneration of injured skeletal muscle after the injury. Muscles Ligaments Tendons. J 2014; 3: 337-345.

23 Arya MA, Tai AK, Wooten EC, Parkin CD, Kudryavtseva E, Huggins GS. Notch pathway activation contributes to inhibition of $\mathrm{C} 2 \mathrm{C} 12$ myoblast differentiation by ethanol. PLoS One. 2013; 8(8): e71632.

24 Crews FT, Bechara R, Brown LA, Guidot DM, Mandrekar P, Oak S, et al. Cytokines and alcohol. Alcohol Clin Exp Res. 2006; 30(4): 720-730.

25 Radek KA, Kovacs EJ, Gallo RL, DiPietro LA. Acute ethanol exposure disrupts VEGF receptor cell signaling in endothelial cells. Am J Physiol Heart Circ Physiol. 2008; 295(1): H174-184.

26 Abou-Khalil R1, Mounier R, Chazaud B. Regulation of myogenic stem cell behavior by vessel cells: the "ménage à trois" of satellite cells, periendothelial cells and endothelial cells. Cell Cycle. 2010; 9(5):892-896.

27 Amaral AC, Parizotto NA, Salvini TF. Dose-dependency of low-energy HeNe laser effect in regeneration of skeletal muscle in mice. Lasers Med Sci. 2001; 16(1): 44-51.

28 Morrone G, Guzzardela GA, Orienti L, Giavaresi G, Fini M, Rocca M, et al. Muscular trauma treated with a Ga-Al-As diode laser: in vivo experimental study. Lasers Med Sci. 1998; 13(4): 293-298.

29 Shefer G, Partridge TA, Heslop L, Gross JG, Oron U, Halevy O. Low-energy laser irradiation promotes the survival and cell cycle entry of skeletal muscle satellite cells. J Cell Sci. 2002; 115(Pt 7): 1461-1469. 